\title{
Ushering in the cardiac role of Ubiquilin1
}

\author{
Xi Fang, Christa Trexler, and Ju Chen
}

Department of Medicine, UCSD, La jolla, California, USA.

Protein quality control (PQC) mechanisms are essential for maintaining cardiac function, and alterations in this pathway influence multiple forms of heart disease. Since heart disease is the leading cause of death worldwide, understanding how the delicate balance between protein synthesis and degradation is regulated in the heart demands attention. The study by $\mathrm{Hu}$ et al. reveals that the extraproteasomal ubiquitin receptor Ubiquilin1 (Ubqln1) plays an important role in cardiac ubiquitination-proteasome coupling, particularly in response to myocardial ischemia/reperfusion injury, thereby suggesting that this may be a new avenue for therapeutics.
The recognition of ubiquitinated proteins by the proteasome

Cardiomyocytes are long-lived, terminally differentiated cells that do not proliferate; thus, they are extremely sensitive to increasing concentrations of misfolded or malfunctioning proteins and rely on an efficient protein quality control (PQC) system to maintain normal cardiac structure and function (1). The first line of defense of the PQC system is the chaperone-cochaperone system, ensuring that proteins are appropriately folded. However, some proteins slip through this checkpoint, leading to accumulation of misfolded proteins $(2,3)$. Furthermore, damaged, oxidized, mutant, and normal proteins that are no longer needed must also be removed in a timely fashion to maintain heart function (1). Thus, the second line of defense of the PQC is the ubiquitin-proteasome system (UPS), which rapidly and effectively recognizes specific substrates to remove misfolded, oxidized, mutant, damaged, or superfluous proteins $(1,4)$. Defects in the UPS occur in multiple cardiac disease settings, underlining the importance of this system in the heart and suggesting that optimizing or augmenting this pathway may have therapeutic potential for cardiac patients (1).
UPS-mediated protein degradation involves two major steps: ubiquitination, whereby a polyubiquitin chain is attached to the target protein via isopeptide bonds, and degradation, whereby ubiquitinated proteins are degraded by the $26 \mathrm{~S}$ proteasome, a multicatalytic protease consisting of a $19 \mathrm{~S}$ regulatory particle (RP) and a $20 \mathrm{~S}$ core particle $(\mathrm{CP})$ that degrades proteins into small oligopeptides (4). The collaborative action of these two steps is crucial for effective PQC. Initial recognition of substrates by the proteasome is mediated by a ubiquitin "tag" on the substrate (4). Two classes of ubiquitin receptors, intraproteasomal and extraproteasomal, recruit "tagged" ubiquitinated proteins to the proteasome (refs. 5, 6, and Figure 1). Intraproteasomal receptors include two 19S proteasomal subunits, Rpn10/S5a and Rpn13/ADRM1, which bind to polyubiquitin via ubiquitin interacting motifs (UIM) $(5,6)$. Extraproteasomal receptors include ubiquitin-like (UBL)/ubiquitin-associated (UBA) proteins that deliver ubiquitinated proteins to the $26 \mathrm{~S}$ proteasome. The UBL/UBA family of proteins contains an $\mathrm{N}$-terminal UBL domain, one or more C-terminal UBA domain or domains, and a variable central region. The UBL domain binds to the UIM motif of Rpn10/S5a pro-

\section{Related Article: p. 5294}

Authorship note: XF and CT contributed equally to this work.

Conflict of interest: The authors have declared that no conflict of interest exists.

Reference information: / Clin Invest. 2018;128(12):5195-5197. https://doi.org/10.1172/JCI124567.

teins in the 19 S proteasome, while the UBA domain binds polyubiquitinated proteins. In a proposed "shuttle-factor" model, UBL/UBA proteins bind to ubiquitinated proteins via the UBA domain and subsequently interact with the proteasome via the UBL domain to ensure that substrates arrive at the proteasome without protein aggregation $(5,6)$.

Ubiquilin (Ubqln)/protein linking integrin-associated protein to cytoskeleton (PLIC) proteins, which are mammalian orthologs of yeast Dsk2, belong to the $\mathrm{UBL} / \mathrm{UBA}$ family $(7,8)$. The Ubiquilin family consists of five paralogous, structurally conserved members that are named UBQLN1 through 4, and UBQLNL (Ubiquilin-like). Each family member harbors an $\mathrm{N}$-terminal UBL domain and a C-terminal UBA domain as well as a stress-inducible heat shock chaperone-binding motif (STI) domain(s) in the middle (7).

The role of Ubiquilin 1 in the heart Thus far, most studies investigating the function of Ubiquilin proteins have focused on the nervous system, as multiple reports have linked Ubiquilins to several neurodegenerative diseases (5). In vivo animal models further demonstrate important functions of Ubiquilin proteins in the brain (9). For example, Ubqln1-overexpressing transgenic mice show increased tolerance to ischemia/ reperfusion (I/R) stress, while Ubqln1 deletion in neurons exacerbates neuronal damage after stroke (9). A potential role of Ubqln1 in diseased heart, however, remains to be addressed. In the current issue of the JCI, Hu and colleagues tackle this important gap in scientific understanding through a variety of novel in vivo and in vitro methods (10) (Figure 1). The authors showed that Ubqln1, which binds a broad range of ubiquitinated substrates, colocalizes with the proteasome in cardiomyocytes and significantly increases in the soluble fraction following I/R stress. Consistent with previous reports in HEK293 cells (11), Ubqln1 was recruited to the ERassociated degradation (ERAD) machin- 
A

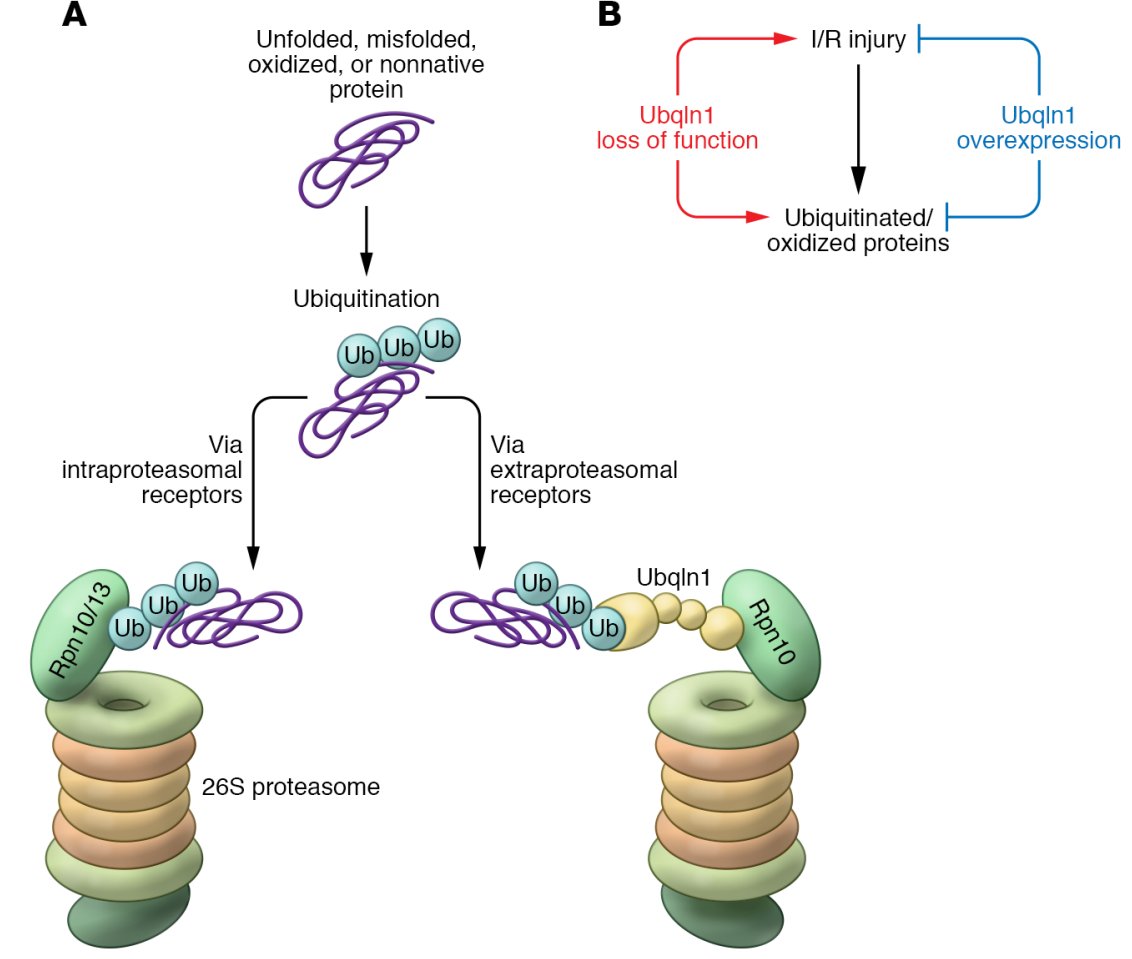

ery in response to ER stress in cardiomyocytes. Cardiomyocyte-specific deletion of Ubqln1 in mice (referred to as CKO mice) resulted in late-onset cardiomyopathy as well as increased accumulation of proteins with lys residues $\mathrm{K} 63-$ and $\mathrm{K} 48-$ linked polyubiquitin chains (Figure 1). The authors also utilized a transgenic mouse line expressing GFPdgn, a well-characterized surrogate substrate of the UPS (9), and observed an accumulation of GFPdgn in the myocardium of Ubqln1-CKO mice. However, activity of proteasomal peptidases was not diminished, demonstrating that coupling between ubiquitination and proteasomal degradation was altered in Ubqln-CKO mice. Together, these observations demonstrate that Ubqln1 plays an essential role in maintaining cardiomyocyte proteostasis.

Importantly, Hu et al. also demonstrated that Ubqln1 deletion in cardiomyocytes exacerbates myocardial I/R injury. Compared with control mice, Ubqln1-CKO mice displayed more severe left ventricular dysfunction as well as larger infarct size following $\mathrm{I} / \mathrm{R}$ injury. Conversely, overexpression of Ubqln1 protected mice from myocardial I/R injury and was associated with improved cardiac function. In vitro experiments performed in isolated cardiomyocytes demonstrated that Ubqln1 overexpression increased the degradation of the UPS surrogate substrate GFPdgn (9) as well as oxidized proteins (Figure 1) without enhancing autophagic flux, suggesting that the protective role of Ubqln1 in response to myocardial I/R injury may be through enhanced clearing of both ubiquitinated and oxidized proteins.

\section{Implications, challenges, and future directions}

This study reveals a novel cardiac role of Ubqln1 and its therapeutic potential in the setting of I/R injury of the heart. Findings in this study raise a number of important questions for future areas of investigation in the field. Characterization of the preference or specificity of substrates targeted by Ubqln1 would help us to fully understand the role of Ubqln1 in cardiomyocytes. The authors showed that Ubqln1 facilitates proteasomal degradation of oxidized proteins and interacts with the ERAD machinery in cultured cardiomyocytes. It would be interesting to determine whether cardiomyocyte-specific deletion of Ubqln1 results in accumulation of oxidized proteins or an enhanced unfolded protein response (UPR) in vivo at baseline or in response to I/R injury. Another issue worth exploring is how Ubqln1 is connected to oxidized protein substrates, ultimately leading to their degradation.
Figure 1. Pathways to transfer ubiquitinated proteins to the proteasome. (A) A ubiquitin tag is added to proteins that need to be degraded, including unfolded, misfolded, nonnative, or oxidized proteins. Two main pathways recruit ubiquitinated proteins to the proteasome: the first relies on intraproteasomal polyubiquitin receptors Rpn10/Rpn13 in the 19S RP, which directly binds polyubiquitin; the second depends upon extraproteasomal ubiquitin receptors, such as UbqIn1 (yellow). UbqIn1 functions as a "shuttle-factor" by binding to ubiquitinated proteins via its UBA domain and subsequently interacts with Rpn10 in the proteasome via its UBL domain. (B) During I/R injury, unfolded, misfolded, and oxidized proteins are increased, putting greater demand on the UPS. Hu et al. reveal that UbqIn1 expression increases with I/R and that loss of UbqIn1 exacerbates, while ubiquitous overexpression of an UbqIn1 transgene ameliorates, myocardial I/R injury. In cultured cardiomyocytes, UbqIn1 overexpression facilitated proteasome-mediated degradation of oxidized proteins.

It is clear that both K48-and K63-linked ubiquitinated proteins are increased in the Ubqln1-CKO heart. Although K48-linked polyubiquitin chains are mostly involved in proteasomal degradation, K63-linked ubiquitination generally serves as a docking site for mediating protein-protein interactions and has diverse proteasome-independent roles (4). Thus, whether accumulated K63-linked polyubiquitin proteins have functional proteasome-independent consequences that may contribute to the observed CKO phenotypes remains to be addressed. In addition to transferring ubiquitinated substrates to the $26 \mathrm{~S}$ proteasome, findings in other cell types suggest that Ubqln 1 is also present within the inner core of aggresomes and functions to deliver ubiquitinated proteins to aggregates (12-14) as well as to regulate aggresome formation via its UBL domain (13). Aggresome formation was not examined by Hu et al.; thus the potential role of Ubqln1 protein in aggresome formation in cardiomyocytes remains to be addressed.

While this work suggests that the main role of Ubqln1 is to promote protein degradation, several studies have shown that Ubqln1 actually stabilizes some of its protein interaction partners, including Presenilin 1/2 (15), BCLb (16), IGF1R (17), ESYT2 (17), IкB $\alpha$ (8), GABA-A (18), and p53 
(19). These observations suggest that, other than potential proteasomal degradation, independent roles of Ubqln1 within the heart also need to be investigated. Furthermore, Ubqln1 can interact with mTOR via its UBA domain; however, this interaction does not seem to have an effect on mTOR turnover, and the function of this interaction is unknown (20). Thus, it is important to consider that loss of Ubqln1 may have consequences independent of protein degradation that may also contribute to the phenotypes observed in the current study.

Finally, the current study has demonstrated a critical role for Ubqln1 in cardiac function during aging and in response to $\mathrm{I} / \mathrm{R}$ injury. It will be of future interest to examine the role of Ubqln1 in the setting of other cardiac disease models. More broadly, this study suggests that impaired ubiquitination-proteasome coupling may represent a major pathogenic risk to the heart, particularly in the context of myocardial I/R injury, and also suggests that improving this coupling may have beneficial therapeutic potential. There are also other extraproteasomal receptor UBL/UBA proteins expressed in the heart (5). It is not clear whether these proteins are altered in Ubqln1-CKO hearts, myocardial I/R, and/or other cardiac diseases. Results of the current study suggest that it will be of interest to examine the potential role of these other UBL/UBA proteins in cardiac function and disease.

\section{Acknowledgments}

Because of space limitations, we were unable to cite many important papers in this field. We would like to thank Sylvia
Evans for constructive suggestions and critical reading of this manuscript. JC is funded by grants from the National Heart, Lung, and Blood Institute and the Foundation Leducq (TNE-13CVD04) and holds an American Heart Association endowed chair in cardiovascular research. XF is supported by NIH K99HL143210.

Address correspondence to: Ju Chen, Department of Medicine, University of California San Diego, 9500 Gilman Drive, BSB 5025, Mail Code 0613C, La Jolla, California 92093, USA. Phone: 858.822.4276; Email:juchen@ucsd.edu.

1. Wang X, Robbins J. Heart failure and protein quality control. Circ Res. 2006;99(12):1315-1328.

2. Young JC, Agashe VR, Siegers K, Hartl FU. Pathways of chaperone-mediated protein folding in the cytosol. Nat Rev Mol Cell Biol. 2004;5(10):781-791.

3. Fang $X$, et al. Loss-of-function mutations in co-chaperone BAG3 destabilize small HSPs and cause cardiomyopathy. J Clin Invest. 2017;127(8):3189-3200.

4. Kleiger G, Mayor T. Perilous journey: a tour of the ubiquitin-proteasome system. Trends Cell Biol. 2014;24(6):352-359.

5. Wang X, Terpstra EJ. Ubiquitin receptors and protein quality control. JMol Cell Cardiol. 2013;55:73-84

6. Finley D. Recognition and processing of ubiquitin-protein conjugates by the proteasome. Annu Rev Biochem. 2009;78:477-513.

7. Lee DY, Brown EJ. Ubiquilins in the crosstalk among proteolytic pathways. Biol Chem. 2012;393(6):441-447.

8. Kleijnen MF, et al. The hPLIC proteins may provide a link between the ubiquitination machinery and the proteasome. Mol Cell. 2000;6(2):409-419.

9. Liu Y, et al. Ubiquilin-1 protects cells from oxidative stress and ischemic stroke caused tissue injury in mice. J Neurosci. 2014;34(8):2813-2821.

10. $\mathrm{Hu} \mathrm{C}$, et al. Inadequate ubiquitinationproteasome coupling contributes to myocardial ischemia-reperfusion injury. J Clin Invest. 2018;128(12):5294-5306.

11. Lim PJ, et al. Ubiquilin and p97/VCP bind erasin, forming a complex involved in ERAD. J Cell Biol. 2009;187(2):201-217.

12. $\mathrm{Kim} \mathrm{SH}$, et al. Potentiation of amyotrophic lateral sclerosis (ALS)-associated TDP-43 aggregation by the proteasome-targeting factor, ubiquilin 1. JBiol Chem. 2009;284(12):8083-8092.

13. Heir R, Ablasou C, Dumontier E, Elliott M, Fagotto-Kaufmann C, Bedford FK. The UBL domain of PLIC-1 regulates aggresome formation. EMBO Rep. 2006;7(12):1252-1258.

14. Regan-Klapisz E, et al. Ubiquilin recruits Eps15 into ubiquitin-rich cytoplasmic aggregates via a UIM-UBL interaction. J Cell Sci. 2005; 118(Pt 19):4437-4450.

15. Mah AL, Perry G, Smith MA, Monteiro MJ. Identification of ubiquilin, a novel presenilin interactor that increases presenilin protein accumulation. J Cell Biol. 2000;151(4):847-862.

16. Beverly LJ, Lockwood WW, Shah PP, Erdjument-Bromage $\mathrm{H}$, Varmus $\mathrm{H}$. Ubiquitination, localization, and stability of an anti-apoptotic BCL2-like protein, BCL2L10/BCLb, are regulated by Ubiquilin1. Proc Natl Acad Sci U S A. 2012;109(3):E119-E126.

17. Kurlawala Z, Shah PP, Shah C, Beverly LJ. The STI and UBA domains of UBQLN1 are critical determinants of substrate interaction and proteostasis. J Cell Biochem. 2017;118(8):2261-2270.

18. Zhang Y, et al. Plic-1, a new target in repressing epileptic seizure by regulation of GABAAR function in patients and a rat model of epilepsy. Clin Sci. 2015;129(12):1207-1223.

19. Feng P, et al. Kaposi's sarcoma-associated herpesvirus $\mathrm{K} 7$ protein targets a ubiquitin-like/ ubiquitin-associated domain-containing protein to promote protein degradation. Mol Cell Biol. 2004;24(9):3938-3948.

20. Wu S, Mikhailov A, Kallo-Hosein H, Hara K, Yonezawa K, Avruch J. Characterization of ubiquilin 1, an mTOR-interacting protein. Biochim Biophys Acta. 2002;1542(1-3):41-56. 